\title{
Identification of dual active agents targeting 5-HT1A and SERT by combinatorial virtual screening methods
}

\author{
Panpan Wang ${ }^{\mathrm{a}}$, Fengyuan Yang ${ }^{\mathrm{a}}$, Hong Yang ${ }^{\mathrm{a}}$, Xiaofei Xu ${ }^{\mathrm{a}}$, Duo Liu ${ }^{\mathrm{b}}$, Weiwei Xue ${ }^{\mathrm{a}}$ and Feng \\ $\mathrm{Zhu}^{\mathrm{a}}{ }^{*}$ \\ ${ }^{a}$ Innovative Drug Research Centre, Chongqing University, Chongqing, 401331, P.R. China \\ ${ }^{b}$ College of Computer Science, Chongqing University, Chongqing, 401331, P.R. China
}

\begin{abstract}
Selective serotonin reuptake inhibitors (SSRIs) are most adopted therapeutics marketed for major depression, and the efficacy of which are greatly reduced by their delayed onset of action and undesirable side effects. 5-HT1A receptor partial agonist and SERT inhibitor (SPARI) was proposed as a novel strategy to overcome the shortage of efficacy by a negative feedback control of 5-HT1A receptor. However, only one SPARI (vilazodone) has been approved for clinical use, and none is currently in clinical trial, which demonstrates a strong need for searching more novel SPARIs to facilitate antidepressants discovery. This work applied a combinatorial virtual screening method (CVSM) by integrating multiple tools. Statistic analysis reveals that CVSM surpasses single virtual screening methods in terms of hit rates and enrichment factors. By adopting optimized CVSM, 91 promising dual target leads form 15 scaffolds were identified, and $40 \%$ of these scaffolds have already been reported to show antidepressant related therapeutic effects. In sum, CVSM is capable in identifying novel SPARIs from large chemical libraries with extremely low false hit rate.
\end{abstract}

Keywords: Virtual screening, support vector machines, molecular docking, 5-HT1A receptor agonist, SERT inhibitor

\section{Introduction}

Depression is one of the most widespread neuropsychiatric diseases. According to the World Health Organization (WHO), depression has become the fourth leading burdensome disease across many countries, and is estimated to be the second life-threatening indication by 2020 [1]. Currently, more than 100 million people were affected by major depression, which severely decrease the quality of people's daily life [2]. Several drugs are now available for major depression, majority of which are selective serotonin reuptake inhibitors (SSRIs) [3]. However, the efficacies of anti-depressants in current market are greatly reduced by their delayed onset of action and undesirable side effects [4]. As reported, negative feedback control of 5-HT1A autoreceptors can decrease the concentration of 5HT on nerve terminal, which further led to a delayed onset of antidepressant [4]. Therefore, multi-target

\footnotetext{
* Address for correspondence: Feng Zhu, Innovative Drug Research Centre, Hu Xi campus, Chongqing University, Higher Education Mega Center in Sha Ping Ba District, Chongqing, P.R. China. Tel.: +86-(0)23-6567-8468; Fax: +86-(0)23-6567-8450; E-mail: zhufeng@cqu.edu.cn.
} 


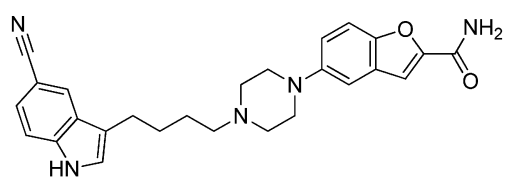

Fig. 1. Chemical structure of vilazodone (5-HT1A: Ki=3 nM; SERT: Ki=5 nM).

drugs simultaneously targeting both 5-HT1A and serotonin transporter (SERT) may propose a novel strategy with enhanced efficacy. In 2011, vilazodone (Figure 1) was approved by FDA as the first 5-HT1A receptor partial agonist and SERT inhibitor (SPARI), which demonstrates a rapid onset of actions and lesser sexual dysfunction [5]. Besides vilazodone, none SPARIs is currently in clinical trial, and only few are in pre-clinical. In order to satisfy the strong need for antidepressants with enhanced efficacy, a new combinatorial virtual screening method (CVSM) is applied in this study to identify novel SPARIs.

\section{Methods}

\subsection{Data collection and support vector machine (SVM)}

Figure 2 illustrates the flowchart of the CVSM used in this study, which is divided into three steps. In step 1, 2,156 and 2,606 active compounds with high affinity $(\leq 500 \mathrm{nM})$ against 5-HT1A and SERT

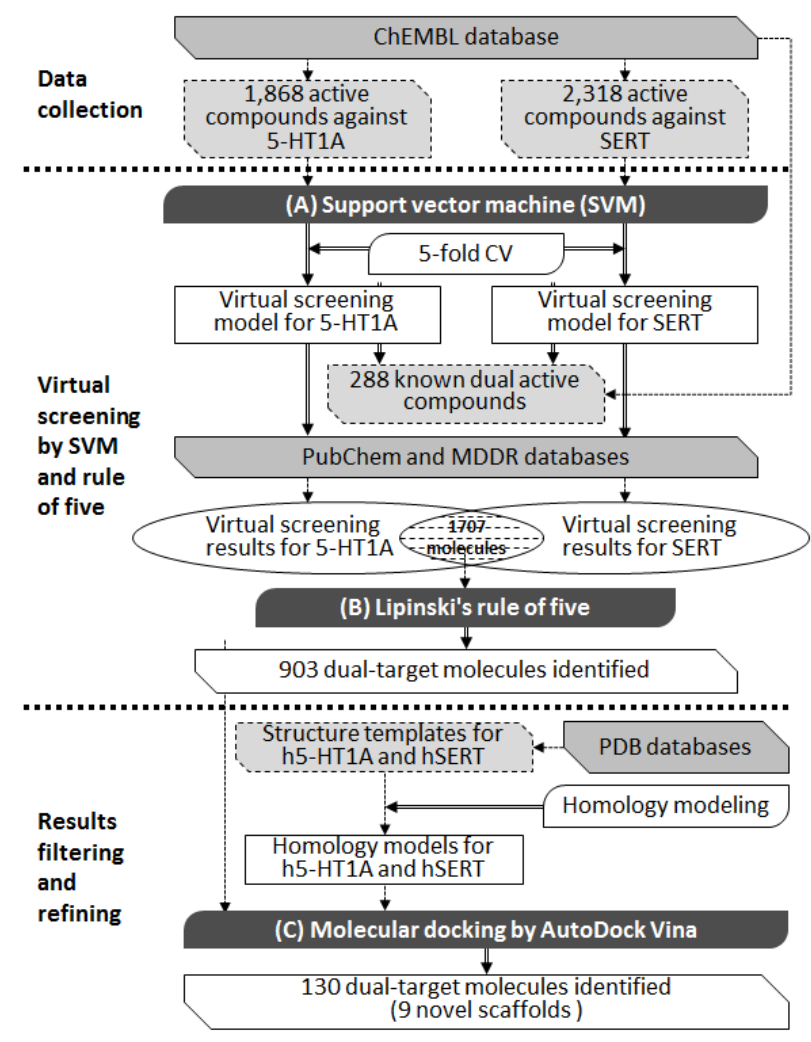

Fig. 2. Flowchart of CVSM in this study. 
were first collected from the ChEMBL database, which include 288 dual active agents. 1,868 and 2,318 single target agents against 5-HT1A and SERT respectively are used as training datasets. Sufficient negative data (non-inhibitors) are vital for reducing false-hits in constructing SVM models [6], but so far only a small amount of them were reported. Thus, putative non-inhibitors were generated using the same method as suggested by Liu, et al [7] to represent non-inhibitor in the whole chemical space. In this work, 62,358 and 61,734 putative non-inhibitors were generated by choosing representatives from families without active compounds respectively. These putative non inhibitors together with individual-target inhibitors constitute the training set for each SVM training model.

Molecular descriptors are quantitative representations of physicochemical features, which have been extensively used in deriving VS tools [7]. In step 2, following the description by Shi, et al [8], a total of 98 molecular descriptors were calculated and SVM models were constructed in this study. VS performance in screening large chemical libraries is measured by several indicators [9], including yield (percentage of known positives predicted as virtual hits), hit-rate (percentage of virtual hits that are known positives), false-hit rate (percentage of virtual hits that are known negatives) and enrichment factor (magnitude of hit-rate improvement over random selection from chemical libraries). In this study, virtual hit and false-hit rate in searching large chemical libraries were evaluated by using 13.56M PubChem and 168K MDDR compounds [8].

\subsection{Homology modeling and molecular docking}

As show in step 3 of Figure 2, molecular docking was used to refine virtual hits identified by SVM and rule of five. Since there is no crystal structural data reported in Protein Data Bank (PDB), the 3D structures of human 5-HT1A and SERT need to be constructed by homology modeling, which was performed by SWISS-MODEL workspace (SMW, http://swissmodel.expasy.org/workspace/). Structural Analysis and Verification Server (SAVES, http://services.mbi.ucla.edu/SAVES/) is further applied to validate the stereo chemical quality of optimized models. Moreover, the energy criteria were checked by Protein Structure Analysis (ProSA, https://prosa.services.came.sbg.ac.at/prosa.php).

Docking program applied here is AutoDock Vina [10]. The active binding sites (Asp116, Val 117, Ile189, Ala203, Phe361, Phe362, Met377) on 5-HT1A were identified by sequence alignment and structure superimposition against its template 4IAR. Moreover, the active binding sites (Try95, Asp98, Ile172, Asn177, Phe341, Ser438) of SERT were resolved from the literature [11]. The grid boxes size were set to $40 * 40 * 40$ points with a grid spacing of $0.375 \AA$ centered on the active sites of 5-HT1A and SERT. All operations of Grid box were performed in Grid module of AutoDock 4.2 [12].

\section{Results and discussion}

\subsection{SVM model construction via 5-fold cross validation}

5-fold cross validation was conducted to test SVM model in identifying known inhibitors. The average accuracies across all 5 folds are $87.79 \%$ and $99.51 \%$ for 5 -HT1A inhibitors and non-inhibitors respectively. For SERT, the corresponding average accuracies are $90.98 \%$ and $99.67 \%$ respectively. The prediction accuracy of single target inhibitors and non-inhibitors are comparable to or better than that of study by Shi [8]. Moreover, prediction accuracy of non-inhibitors statistically indicates a very low false-hit rate, which is very important for further validation. In sum, SVM models constructed in this work showed good prediction capabilities for identifying known inhibitors. 


\subsection{Independent test and virtual screening of large compound libraries}

By combining SVM virtual screening models of 5-HT1A and SERT, 142 out of 288 (49.31\%) dual active compounds collected from ChEMBL were successfully identified, which is slightly superior to the reported $47.7 \%$ of another VS result [8]. Among those 288 compounds, 2 out of $19(10.53 \%)$ compounds outside the common training active families of both targets were correctly predicted, which shows certain level of capacity in identifying novel dual target inhibitors.

In addition to good hit rates in both 5-fold cross validation and independent test, the constructed SVM model shows a substantially low false-hit rate. 28,141 compounds are identified as active which represent only $0.208 \%$ of $13.56 \mathrm{M}$ PubChem compounds, and the estimated false-hit rate in screening 168K MDDR compounds is only 1.016\% (1,707 identified hits). Moreover, all 11 inactive compounds against both targets from ChEMBL were correctly predicted by our methods, which suggested a good capacity of SVM in distinguishing active dual target agent from inactive ones.

\subsection{Homology modeling and validation}

Templates used for homology modeling were generated by the automated mode of SWISS-MODEL workspace (SMW). By aligning human protein sequences against PDB database, drosophila melanogaster dopamine transporter chain A (PDB ID: 4M48) and human chimeric protein 5-HT1B-BRIL (PDB ID: 4IAR) were found with the highest sequence identities to hSERT and h5-HT1A (53.37\% and 45.33\% respectively). SWISS-PDB viewer from SMW was further applied to minimize the overall energy of both models.

In order to validate the constructed models, Structural Analysis and Verification Server (SAVES) were utilized. In this study, we used three modules to evaluate the stereo chemical quality of the constructed models (PROCHECK, VERIFY 3D and ERRAT). PROCHECK conducted the Ramachandran plot analysis and identified $88.3 \%$ and $88.9 \%$ residues of 5-HT1A and SERT as within the most favored regions, while only $0.3 \%$ and $0.2 \%$ residues are within the disallowed regions. This validates a good quality of each optimized model [13]. Moreover, SERT and 5HT-1A models are verified as "pass" and "warning" by VERIFY 3D with $83.36 \%$ and $67.01 \%$ residues of preferred VERIFY 3D-1D score $(\geq 0.2)$. By comparing 5 HT-1A model with its corresponding template, the percentages of residues with preferred 3D-1D score are comparable to each other $(67.01 \%$ to $76.25 \%)$, which validate the relatively low percentage of 5HT-1A model. Furthermore, the overall quality factor ERRAT were 84.759 and 87.430 for 5-HT1A and SERT respectively, which could be considered as high quality models [14].

Moreover, model quality and the deviation of structure total energy with respect to random conformation energy were evaluated by Z-score from the Protein Structure Analysis (ProSA). Z-scores of 5-HT1A, 5-HT1A template, SERT and SERT template were -5.56, -4.52, -5.34 and -6.54 respectively. Scores between protein and its template are in the range of similar size proteins, which indicates reliable modeling structures.

\subsection{Refinement of virtual screening results by rule of five and molecular docking}

To enhance the hit rate of virtual screening, 1,849 molecules (1,707 from MDDR and 142 known dual active compound from ChEMBL) predicted by SVM were further filtered by rule of five and then refined by molecular docking. A statistic comparison among various combinatorial virtual screening methods was shown in Table 1. Comparing with SVM, the CVSM demonstrate a significant 
Table 1

Comparison of yield, hit rate and enrichment factor among various combinatorial virtual screening methods

\begin{tabular}{llllll}
\hline Method & Predicted inhibitors & Hits & Yield (\%) & Hit rate (\%) & Enrichment factor \\
\hline SVM & 1,849 & 142 & $49.31 \%$ & $7.68 \%$ & 44.86 \\
SVM+R5 & 1,043 & 140 & $48.61 \%$ & $13.42 \%$ & 78.41 \\
SVM+R5+Docking Top 0.3\% & 504 & 75 & $26.04 \%$ & $14.88 \%$ & 86.93 \\
SVM+R5+Docking Top 0.2\% & 336 & 71 & $24.65 \%$ & $21.13 \%$ & 123.44 \\
SVM+R5+Docking Top 0.1\% & 168 & 38 & $13.19 \%$ & $22.62 \%$ & 132.14 \\
\hline
\end{tabular}

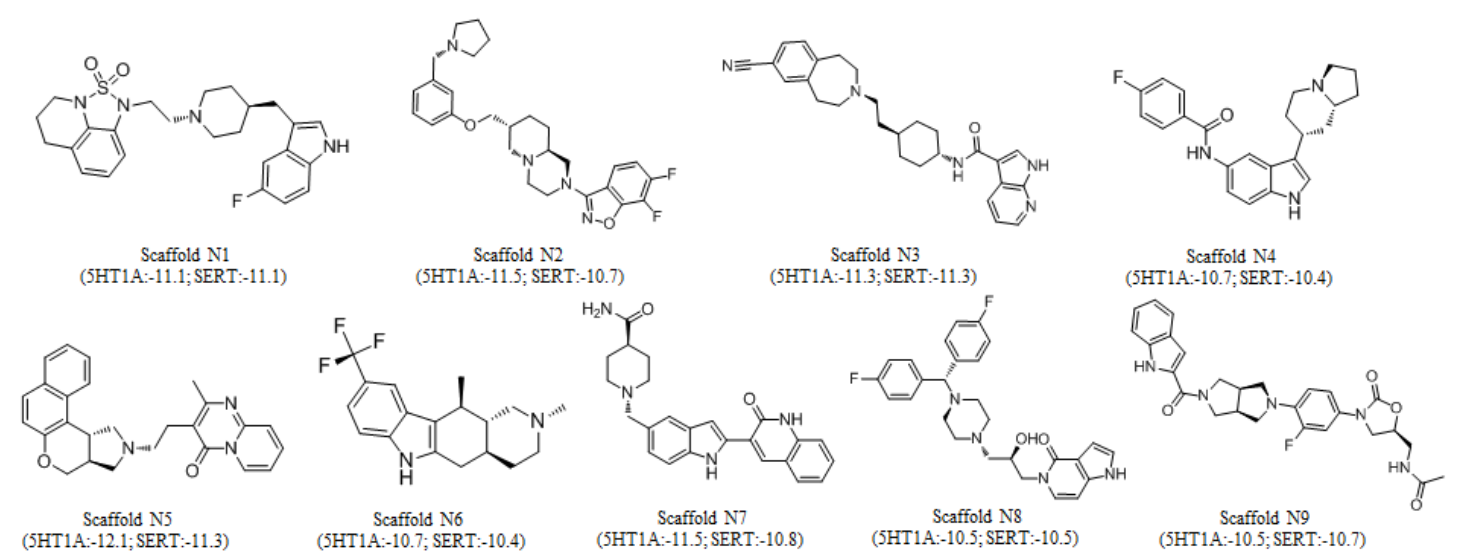

Fig. 3. 9 examples of molecular scaffolds identified as novel SPARIs in this work (affinity (kcal/mol) predicted by Vina for 5-HT1A and SERT were shown in the parentheses).

increase in both hit rates (from $7.68 \%$ to $22.62 \%$ ) and enrichment factors (44.86 to 132.14). Moreover, with the increases of docking cut-off (from top $0.3 \%$ to $0.1 \%$ ), the enrichment factors of CVSM increase significantly from 86.93 to 132.14 (see Table 1).

In order to maximize the enrichment, Top $0.1 \%$ was selected as the final screening cut-off, which results in 168 identified candidates (130 out of 168 are unknown dual active compounds identified by this work). Majority of these identified candidates (91 out of 130) are ranked higher than vilazodone by Vina (its predicted affinities are -10.5 and $-10.3 \mathrm{kcal} / \mathrm{mol}$ for $5-\mathrm{HT} 1 \mathrm{~A}$ and SEAR respectively).

These 91 molecules covered 15 scaffolds, 24 of which are structurally dissimilar to known inhibitors. Figure 3 illustrates 9 novel scaffolds identified in this study, 6 of these scaffolds have already shown antidepressant therapeutic effects. LY367265 of Scaffold N1 (indole derivatives) was a potential antidepressant drug targeted 5HT2A and SERT, and the affinity Ki is $490 \mathrm{nM}$ in rat cerebral cortex [15]. Scaffold N2 (2- (benzo[d]isoxazol-3-yl)-7- (phenoxymethyl) octahydropyrido [1,2-a] pyrazine) was used for psychosis therapy as agonists or antagonists of serotonin 1A or 1D receptors, and was pointed out that they may be potentially useful for treating a wide variety of conditions co-administered with 5-HT reuptake inhibitors [16]. Scaffold N3 (tetrahydrobenzazepine derivatives) was an antipsychotic dopamine (D3) Antagonist [17]. Scaffold N4 (substituted heteroaromatic octahydroindolizinyl indoles) was reported to have valuable pharmacological properties, especially to treat migraine and associated disorders as 5-HT1F Agonist [18]. Scaffold N5 (pyrimidin-4-one derivatives) was reported to treat disorders such as depression, impulsive behavior, anxiety, schizophrenia, Parkinson's disease, cognition disorder, libido disorder, sexual dysfunction, appetite disorder and sleep disorder as $\alpha 2 / 5-\mathrm{HT} 2 \mathrm{C}$ double antagonists [19]. Scaffold N6 (fused indole derivatives) are $\mathrm{MCH}$ receptor modulators used in obesity, anxiety and mood disorders therapy [20]. 

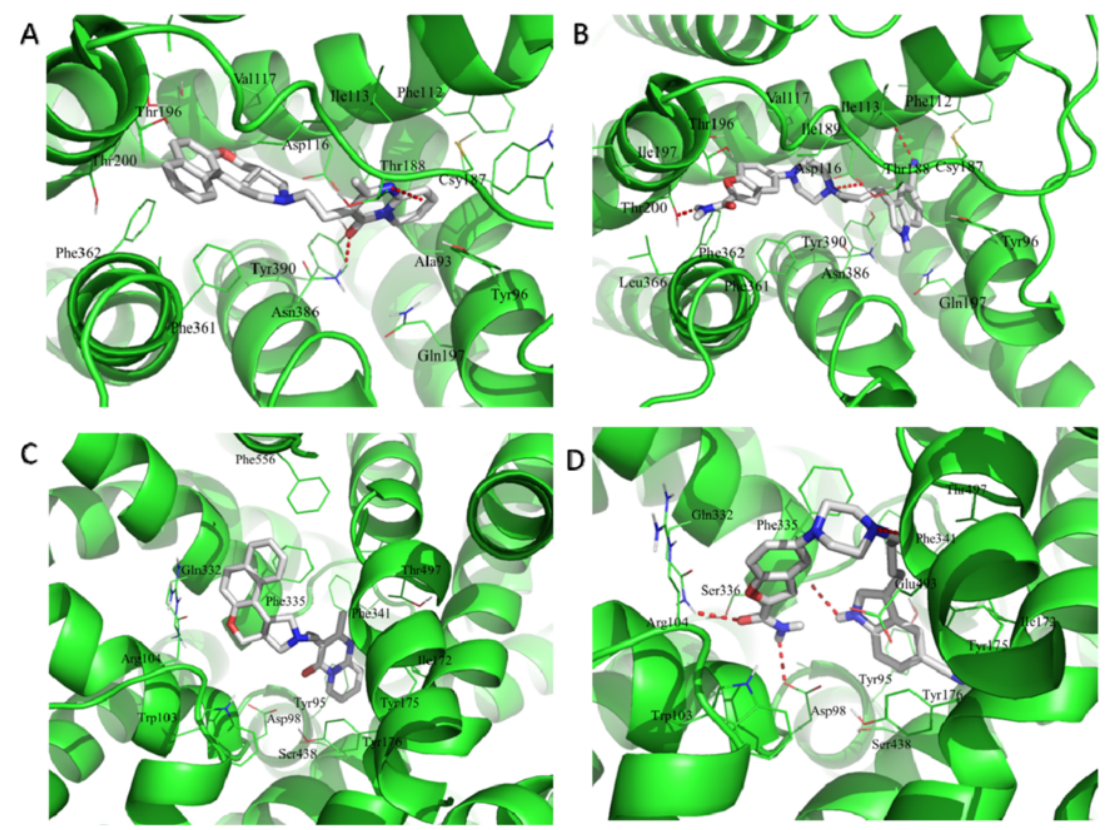

Fig. 4. Molecular docking of scaffold N5 and vilazodone with target proteins (Hydrogen bonds are labeled as red dotted lines): (A) scaffold N5 with 5-HT1A; (B) vilazodone with 5-HT1A; (C) scaffold N5 with SERT; (D) vilazodone with SERT.

In addition, docking results reveal that the 9 identified novel compounds bind to each targets have similar mode with vilazodone. Figure 4 gives the binding poses of scaffold N5 and vilazodone in the drug binding domain of 5-HT1A and SERT. In detail, Scaffold N5 and vilazodone primarily interacted with Asp116, Csy187, Leu366 and Tyr390 of 5-HT1A (Figure 4A and 4B ), and the binding pocket for Scaffold N5 and vilazodone in SERT were formed by residues Try95, Asp98, Trp103, Arg104, Ile172, Tyr175, Tyr176, Gln332, Phe335, Phe341, Ser438, Thr497 (Figures 4C and 4D).

\section{Conclusion}

Combinatorial virtual screening methods integrating SVM, RO5 and molecular docking were used to identify dual active compounds against both 5-HT1A and SERT in this study. By screening large chemical library MDDR, SVM identified 1,707 compounds from MDDR database with a extremely low false hit rate (1.016\%). By combining rule of 5 and molecular docking, 9 novel scaffolds with significantly enhanced enrichment factors were identified, some of which have already shown antidepressant related effects, which raises urgent needs on conducting more comprehensive screening of an even larger chemical space to identify more SPARIs.

\section{Acknowledgment}

We gratefully acknowledge the research support of the National Natural Science Foundation of China (81202459), the Fundamental Research Funds for the Central Universities (CDJKXB14005, CDJZR14468801 and CDJZR14185501) and the Chongqing Graduate Student Research Innovation 
Project (CYB14027).

\section{References}

[1] J. Prins, B. Olivier and S.M. Korte, Triple reuptake inhibitors for treating subtypes of major depressive disorder: The monoamine hypothesis revisited, Expert Opinion on Investigational Drugs 20 (2011), 1107-1130.

[2] J.A. Reinhold, L.A. Mandos, F.W. Lohoff and K. Rickels, Evidence for the use of vilazodone in the treatment of major depressive disorder, Expert Opinion on Pharmacotherapy 13 (2012), 2215-2224.

[3] M.S. Stephen, L.Z. Clara, C. Sylvia and A.M. Debbi, Serotonergic drugs for depression and beyond, Current Drug Targets 14 (2013), 578-585.

[4] K.R. Starr, G.W. Price, J.M. Watson, P.J. Atkinson, R. Arban, S. Melotto, L.A. Dawson, J.J. Hagan, N. Upton and M.S. Duxon, SB-649915-B, a novel 5-HT1A/B autoreceptor antagonist and serotonin reuptake inhibitor, is anxiolytic and displays fast onset activity in the rat high light social interaction test, Neuropsychopharmacology 32 (2007), 2163-2172.

[5] M. Singh and T.L. Schwartz, Clinical utility of vilazodone for the treatment of adults with major depressive disorder and theoretical implications for future clinical use, Neuropsychiatric Disease and Treatment 8 (2012), 123-130.

[6] B.N. Chen, R.F. Harrison, G. Papadatos, P. Willett, D.J. Wood, X.Q. Lewell, P. Greenidge and N. Stiefl, Evaluation of machine-learning methods for ligand-based virtual screening, Journal of Computer-Aided Molecular Design 21 (2007), $53-62$.

[7] X.H. Liu, X.H. Ma, C.Y. Tan, Y.Y. Jiang, M.L. Go, B.C. Low and Y.Z. Chen, Virtual screening of Abl inhibitors from large compound libraries by support vector machines, Journal of Chemical Information and Modeling 49 (2009), 2101-2110

[8] Z. Shi, X.H. Ma, C. Qin, J. Jia, Y.Y. Jiang, C.Y. Tan and Y.Z. Chen, Combinatorial support vector machines approach for virtual screening of selective multi-target serotonin reuptake inhibitors from large compound libraries, Journal of Molecular Graphics and Modelling 32 (2012), 49-66.

[9] H. Li, C.W. Yap, C. Ung, Y. Xue, Z.R. Li, L.Y. Han, H.H. Lin and Y.Z. Chen, Machine learning approaches for predicting compounds that interact with therapeutic and ADMET related proteins, Journal of pharmaceutical sciences 96 (2007), 2838-2860.

[10] O. Trott and A.J. Olson, AutoDock Vina: improving the speed and accuracy of docking with a new scoring function, efficient optimization, and multithreading, Journal of Computational Chemistry 31 (2010), 455-461.

[11] L. Sørensen, J. Andersen, M. Thomsen, S.M. Hansen, X. Zhao, A. Sandelin, K. Strømgaard and A.S. Kristensen, Interaction of antidepressants with the serotonin and norepinephrine transporters mutational studies of the s1 substrate binding pocket, Journal of Biological Chemistry 287 (2012), 43694-43707.

[12] R.J. Rosenfeld, D.S. Goodsell, R.A. Musah, G.M. Morris, D.B. Goodin and A.J. Olson, Automated docking of ligands to an artificial active site: augmenting crystallographic analysis with computer modeling, Journal of Computer-Aided Molecular Design 17 (2003), 525-536.

[13] V. Hooda, P. babu Gundala and P. Chinthala, Sequence analysis and homology modeling of peroxidase from Medicago sativa, Bioinformation 8 (2012), 974-979.

[14] A. Messaoudi, H. Belguith and J.B. Hamida, Homology modeling and virtual screening approaches to identify potent inhibitors of VEB-1 $\beta$-lactamase, Theoretical Biology and Medical Modelling 10 (2013), 22-32.

[15] I.A. Pullar, S.L. Carney, E.M. Colvin, V.L. Lucaites, D.L. Nelson and S. Wedley, LY367265, an inhibitor of the 5-hydroxytryptamine transporter and 5-hydroxytryptamine $2 \mathrm{~A}$ receptor antagonist: a comparison with the antidepressant, nefazodone, European Journal of Pharmacology 407 (2000), 39-46.

[16] B.G. Michael, Azabicyclic 5-HT1 receptor ligands, patent WO 9952907 A1, Jan. 1999.

[17] J.C.N. Hadley, Michael Stewart MacDonald, Gregor James, Preparation of tetrahydrobenzazepine derivatives as modulators of dopamine D3 receptors (antipsychotic agents), patent WO 2000021951 A1, Apr. 2000.

[18] K.J.H. Filla, A. Sandra and Schaus John Mehnet, Substituted heteroaromatic 5-HT1F agonists, including (octahydroindolizinyl)indoles and analogs, useful as antimigraine agents, patent US 5874427 A, Feb. 1999.

[19] D.T. La Vielle and Muller Olivier Gillert, Preparation of pyrimidin-4-one derivatives, their pharmaceutical compositions and use as $\alpha 2 / 5$-HT2c double antagonists, patent EP 1256583, Oct. 2002.

[20] X.Q. Chen, K. Dai and P.C. Fan, Preparation of fused indole derivatives as MCHR modulators for treatment of obesity, patent WO 2002089729 A2, Nov. 2002. 